\title{
Oxytocin modulates proliferation and stress responses of human skin cells: implications for atopic dermatitis
}

\author{
Verena Deing ${ }^{1}$, Dennis Roggenkamp ${ }^{1}$, Jochen Kühnl' ${ }^{1}$ Alisa Gruschka' ${ }^{1}$ Franz Stäb ${ }^{1}$, Horst Wenck ${ }^{1}$, \\ Alexander Bürkle ${ }^{2}$ and Gitta Neufang ${ }^{1}$ \\ ${ }^{1}$ Beiersdorf AG, Research Skin Care, Hamburg, Germany; ${ }^{2}$ Molecular Toxicology, University of Konstanz, Konstanz, Germany \\ Correspondence: Gitta Neufang, PhD, Beiersdorf AG, Research Skin Care, Unnastraße 48, 20245 Hamburg, Germany, Tel.: +49 40 4909 6676, Fax: \\ +49404909 186676, e mail: gitta.neufang@beiersdorf.com
}

\begin{abstract}
The neuropeptide hormone oxytocin (OXT) mediates a wide spectrum of tissue specific actions, ranging from cell growth, cell differentiation, sodium excretion to stress responses, reproduction and complex social behaviour. Recently, OXT expression was detected in keratinocytes, but expression of its receptor and function are still unexplored in human skin. Here, we showed that both OXT and its receptor are expressed in primary human dermal fibroblasts and keratinocytes. OXT induced dose dependent calcium fluxes in both cell types demonstrating that the OXT receptor (OXTR) is functionally expressed. We also showed that OXT decreases proliferation of dermal fibroblasts and keratinocytes in a dose dependent manner. In order to further investigate OXT mediated functions in skin cells, we performed OXTR knockdown experiments. OXTR knockdown in dermal fibroblasts and keratinocytes led to elevated
\end{abstract}

levels of reactive oxygen species and reduced levels of glutathione (GSH). Moreover, OXTR depleted keratinocytes exhibited an increased release of the pro inflammatory cytokines IL6, CCL5 and CXCL10. Our data indicate that the OXT system modulates key processes which are dysregulated in atopic dermatitis (AD) such as proliferation, inflammation and oxidative stress responses. Furthermore, we detected a downregulation of the OXT system in peri lesional and lesional atopic skin. Taken together, these data suggest that the OXT system is a novel neuroendocrine mediator in human skin homoeostasis and clinically relevant to stressed skin conditions like $\mathrm{AD}$.

Key words: atopic dermatitis - inflammation - oxidative stress oxytocin system - proliferation

kinase A pathway, whereas mitogenic effects are associated with an increase of intracellular calcium and tyrosine phosphorylation (14). Further functions of OXT are the modulation of neuroendo crine stress responses and inflammatory processes $(18,19)$. OXT dampens the hypothalamo pituitary adrenal (HPA) axis by lower ing corticosterone/cortisol levels in response to acute exposure to stressors $(18,20)$. Human skin takes part in the local as well as the systemic neuroendocrine network (21). For instance, all key medi ators of the HPA axis including the neuropeptides $\mathrm{CRH}$, POMC derived ACTH, $\alpha \mathrm{MSH}, \beta$ endorphin and cortisol/corticosterone are synthesized by skin cells (21 23). In term myometrium, CRH receptor function is inhibited by OXT via activation of protein kinase $\mathrm{C}$ (24). In view of possible cross talks, CRH receptors are also expressed by dermal fibroblasts and keratinocytes $(22,25,26)$. However, with respect to inflammation, subcutaneously given OXT has been demonstrated to prevent sepsis induced depletion of glutathione (GSH) contents in colonic and uterine tissues of rats (27). Furthermore, wound healing, which involves a well organized inflammatory phase, has been shown to be facilitated by OXT administration $(28,29)$. Moreover, in a rat model of dried latex induced paw oedema, OXT treatment showed anti inflammatory and antinociceptive activity, thus pointing to a role of OXT in skin inflammation (30). Inflammation is typically accompanied by oxi dative stress. In an in vitro model, OXT decreased not only IL6 secretion, but also NADPH dependent superoxide production in vascular cells and THP 1 macrophages suggesting that OXT attenu ates vascular oxidative stress (31).

In humans, chronically inflamed skin is, amongst others, evident in atopic dermatitis (AD). The skin of patients suffering 
from $\mathrm{AD}$ shows lichenification, erythema and pruritic lesions. $\mathrm{AD}$ is a multifactorial skin disease: genetic predisposition, bacterial infections, skin barrier disruption, immunological alterations and psychological stress have all been discussed as causes and aggravation factors $(32,33)$. Epidermal hyperplasia is a common feature of $\mathrm{AD}$ and attrib uted to $\mathrm{T}$ cell derived cytokines such as tumor necrosis factor $\alpha$ and interferon $\gamma$ which are potent inducers of epidermal growth factors and their receptor (34 36). Additionally, these cytokines initiate inflammatory cascades and have been shown to induce an abnormal chemokine production profile in keratinocytes of $\mathrm{AD}$ patients (37).

A recent study showed that in human skin, OXT is also produced by epidermal keratinocytes (38). However, its role in cutaneous homoeostasis remains unknown. The aim of the present study was the characterization of the OXT system in human skin and the analysis of its functional activity and relevance in skin physiology. We showed that dermal fibroblasts and keratinocytes express OXT and its receptor. Additionally, we revealed the role of OXT in the modulation of cutaneous proliferation, inflammation and oxidative stress responses with possible implications for AD.

\section{Methods}

\section{In vivo studies}

All in vivo studies were conducted according to the Declaration of Helsinki and approved by the ethics committees of the Medical Associations at Hamburg, Kiel and Freiburg, Germany. All study participants gave written informed consent. AD patients displayed a local SCORAD of $5.8 \pm 0.3$ on test arm area. Ages of healthy $(n=32)$ and $\mathrm{AD}(n=24)$ participants were equivalent (healthy: $36.6 \pm 2.1$, atopic: $35.0 \pm 1.7$ years). Punch biopsies were taken under local anaesthesia from peri lesional/lesional areas of the forearm of atopic patients and healthy volunteers. Suction blisters were prepared as described by Kiistala (39). In an additional study, suction blister fluids were taken from the inner upper arms of six healthy women and six healthy men aged 2040 at $t_{0}$ and $t_{1}$. At $t_{1}$, participants caressed themselves with a soft brush every $30 \mathrm{~min}$ for 5 min during $34 \mathrm{~h}$ around the investigated area (tactile stimulation) leaving the other arm unstimulated (control).

\section{Isolation and culture of primary human dermal fibroblasts} and keratinocytes

Skin cells were isolated from biopsies and cultured as described by Roggenkamp et al. (40), and used at passages 1 5. As determined by ELISA, basal OXT concentrations in culture medium for fibro blasts and keratinocytes were $66 \mathrm{pm}$ and $2.7 \mathrm{nM}$, respectively. Physiological plasma OXT levels range from 5 to 300 рм (41 43). In knockdown experiments, no additional OXT was applied. OXT doses used in the experiments to determine $\mathrm{Ca}^{2+}$ fluxes and prolif eration have been previously elaborated (44).

Immunofluorescence analyses

Cells grown on glass chamber slides (Thermo Fischer Scientific, Waltham, MA, USA) were fixed with $4 \%$ paraformaldehyde (Sigma Aldrich, Munich, Germany) in PBS and permeabilized with $0.1 \%$ Triton X 100 (Sigma Aldrich) in PBS. Cryosections of human skin were fixed with ice cold acetone. Cells and sections were blocked with 1\% BSA in PBS and incubated with antibodies directed against human OXT (fibroblasts, keratinocytes: 1:200, LS C37953; LifeSpan BioSciences (Seattle, WA, USA), skin: 1:500, MAB5296; Millipore, Billerica, MA, USA) and OXTR (1:200, SYC592; Eurogentec, Seraing, Belgium). For staining of nuclei and visualiza tion, cells and sections were incubated with Alexa 488 conjugated secondary antibodies (1:1000; Invitrogen, Darmstadt, Germany) mixed with DAPI (1:2000, $5 \mathrm{mg} / \mathrm{ml}$ stock; Sigma Aldrich). Micros copy was performed with an Axiovert S100 (Zeiss, Jena, Germany).

\section{Real time semi quantitative PCR}

Total RNA from fibroblasts, keratinocytes and suction blister derived epidermis was isolated using RNeasy Kit (Qiagen, Hilden, Germany). Concentration and purity of RNA were assessed utiliz ing a NanoDrop 1000 (Peqlab, Erlangen, Germany). RNA was subjected to cDNA synthesis with the high capacity cDNA Reverse Transcription Kit (Applied Biosystems, Darmstadt, Germany). Expression levels were detected by semiquantitative real time poly merase chain reaction (RT PCR) using gene expression assays mixed with TaqMan Master Mix performed in a 7900HT Fast RT PCR System (all from Applied Biosystems). The gene expression assays Hs00792417 g1 (OXT) and Hs00168573 m1 (OXTR) were utilized. Expression was quantified by the compara tive $\Delta C_{\mathrm{t}}$ method using $18 \mathrm{~S}$ expression as endogenous control.

\section{Detection of oxytocin and cytokine protein levels}

OXT concentrations in suction blister fluids were measured using an OXT ELISA (Enzo Life Sciences, Lörrach, Germany) and nor malized to total protein contents determined by BCA quantifica tion (Interchim, Montlucon, France). Cytokine concentrations were measured using a 27 Plex (BioRad, Munich, Germany).

\section{Oxytocin receptor knockdown}

One day after seeding cells at a density of $2 \times 10^{5} /$ well into six well plates, they were transfected with $20 \mathrm{~nm}$ (fibroblasts) or $50 \mathrm{~nm}$ (keratinocytes) of scrambled (No.1027281; Qiagen) or OXTR specific (AM16708, ID1766; Invitrogen) siRNA using Lipofectamine RNAiMAX (Invitrogen) according to the manufacturer's instructions. Measurement of $\mathrm{Ca}^{2+}$ currents

Intracellular calcium concentrations were measured by flow cytometry as described previously (45). Briefly, cells were incu bated with PBS containing $3 \mu \mathrm{g} / \mathrm{ml}$ Fluo $4 \mathrm{AM}, 10 \mu \mathrm{g} / \mathrm{ml}$ Fura Red, 0.02\% Pluronic Detergent F 127 and $4 \mathrm{~mm}$ Probenecid and $10 \%$ FCS (all from Invitrogen). $\mathrm{Ca}^{2+}$ currents were induced by OXT (Tocris, Bristol, UK) diluted in 10\% FCS in PBS and recorded using the flow cytometer FACSCanto ${ }^{\mathrm{TM}}$ (BD Bioscience, Heidelberg, Germany). Inhibition of OXT induced $\mathrm{Ca}^{2+}$ currents was performed by pre addition of the OXTR specific antagonist L371,257 (Tocris). Data were analysed using FlowJo 7.2.1 (BD Bioscience). $\mathrm{Ca}^{2+}$ currents were normalized to cell number. Imaging of $\mathrm{Ca}^{2+}$ fluxes was carried out using the confocal microscope SP5 (Leica, Bensheim, Germany).

\section{Proliferation assay}

Fibroblasts and keratinocytes were seeded in 96 well plates. One day after plating, cells were treated daily with OXT alone or in combination with the OXTR specific antagonist L371,257. At day 4 and 7 after initial treatment, cells were fixed and stained with $6.2 \mu \mathrm{M}$ propidium iodide (46) (Invitrogen) and 17\% ethanol in PBS. Prolifer ation was quantified by cell counting using an Axiovert 200M (Zeiss).

\section{UV irradiation}

For UV exposure (47), cells grown in six well plates were irradi ated in $1 \mathrm{ml} \mathrm{PBS/well.} \mathrm{Fibroblasts} \mathrm{were} \mathrm{irradiated} \mathrm{with} 5 \mathrm{~J} / \mathrm{cm}^{2}$ UVA light using a Psorisan 900/5164 H1 (Dr. Hönle Medizintech nik, Kaufering, Germany). Keratinocytes were irradiated with solar simulated light (SSR) using an Oriel 1600W (Newport Corpora tion, Stratford, CT, USA) at a dose of $100 \mathrm{~mJ} / \mathrm{cm}^{2}$ UVB. UV doses were determined with an IL1700 Research Radiometer (Interna tional light, Newburyport, MA, USA). The UV spectra of the light 
sources used are provided as supplemental figures (Figs S1 and S2). Directly after irradiation, cells were subjected to reactive oxy gen species (ROS) and GSH determination assays.

Determination of reactive oxygen species

Cells were incubated with $150 \mathrm{ng} / \mathrm{ml} \mathrm{2} 2^{\prime}, 7^{\prime}$ dichlorodihydrofluores cein diacetate (Invitrogen) in PBS according to the manufacturer's instructions. Fluorescence intensities were measured at $535 \mathrm{~nm}$ using a Safire reader (Tecan, Crailsheim, Germany) and normal ized to cell number.

Detection of glutathione

Cells were incubated with $20 \mu \mathrm{M}$ ThiolTracker ${ }^{\mathrm{TM}}$ Violet dye (Invi trogen) in PBS according to the manufacturer's instructions. This dye was used to estimate the cellular level of reduced GSH, because it represents the majority of intracellular free thiols in the cell. Fluorescence intensities were measured at $526 \mathrm{~nm}$ using a Safire reader (Tecan) and normalized to cell number.

Statistics

Data were analysed with Prism 5 (GraphPad Software, San Diego, CA, USA) and are presented as the mean \pm SEM. Normality was checked by the Shapiro Wilk test. If the hypothesis of normality was rejected, groups were compared using the Mann Whitney $U$ test (unpaired data) or the Wilcoxon signed rank test (paired data). Otherwise, Studen's $t$ test (paired/unpaired data) was used for group comparison. $P$ values $<0.05$ were considered statistically significant.

\section{Results}

The oxytocin system is functionally expressed in primary human skin cells

Oxytocin expression in keratinocytes has recently been reported (38), but expression of the OXT receptor (OXTR) and its role in human skin have not been investigated so far. Immunofluorescence analyses revealed that both OXT and the OXTR are expressed in dermal fibroblasts and keratinocytes (Fig. 1a d). Furthermore, OXT is localized throughout the epidermis (Fig. le). In contrast, the OXTR is mainly detected in the basal layer of the epidermis (Fig. 1f). RT PCR analyses confirmed OXT and OXTR expression in skin cells (Fig. 1g,h). The highest mRNA expression levels of OXT and the OXTR were detected in fibroblasts compared to keratinocytes or epidermis tissue. OXT expression in keratinocytes was eightfold higher than in the epidermis. This was presumably due to a higher expression of OXT in proliferating keratinocytes (38), which were pre dominant in the in vitro monoculture, compared to differentiated keratinocytes, predominant in the epidermis in vivo/ex vivo. Hypothal amus mRNA served as positive control for OXT/OXTR expression.

To examine OXT protein levels in human skin in vivo, we analysed suction blister fluids, taken from both arms of 12 healthy volunteers. With respect to gender, OXT levels were equivalent (males: $6.5 \pm 0.6 \mathrm{pg}$ OXT/mg protein, females: $6.4 \pm 1 \mathrm{pg}$ OXT/mg pro tein) (Fig. 1i). As higher OXT concentrations are found in the saliva upon tactile stimulation (48), we investigated whether OXT is also released locally by tactile stimulation from skin tissue. To this end, suction blister fluids were taken either after repeated tactile stim ulation or from the corresponding, unstimulated arm. Significantly higher OXT concentrations were observed in suction blister fluids taken from stimulated arms $(6.8 \pm 0.7 \mathrm{pg}$ OXT/mg protein) com pared to unstimulated arms $(5.9 \pm 0.4 \mathrm{pg} \mathrm{OXT} / \mathrm{mg}$ protein) (Fig. 1j), indicating a local OXT release from tactile stimulated skin.

OXTRs predominantly couple to $\mathrm{G}_{\mathrm{q}}$ proteins and enhance ino sitol 3 phosphate signalling, leading to increased intracellular $\mathrm{Ca}^{2+}$ (a)

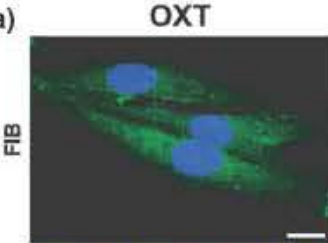

(c)

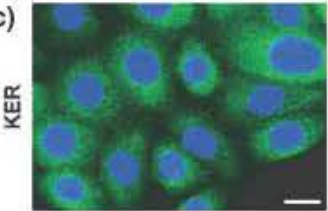

(e)
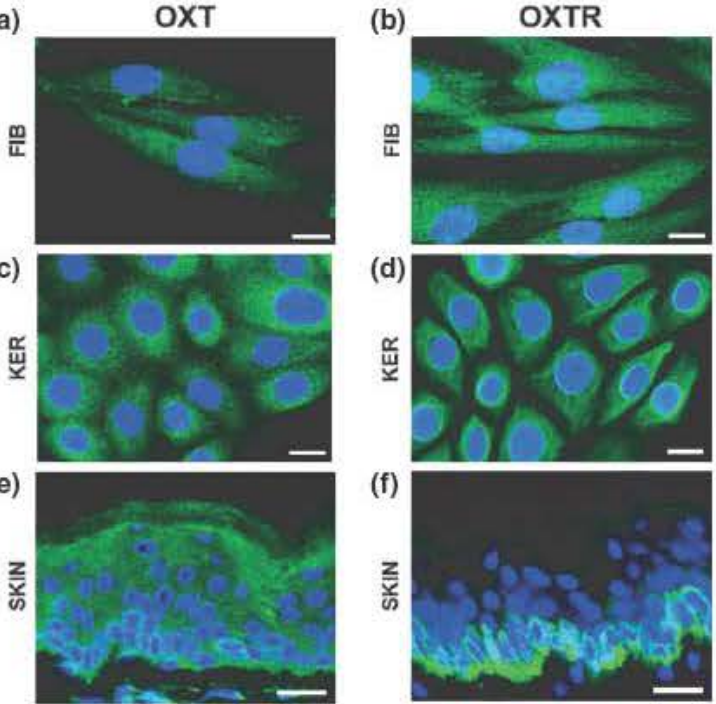

(d)
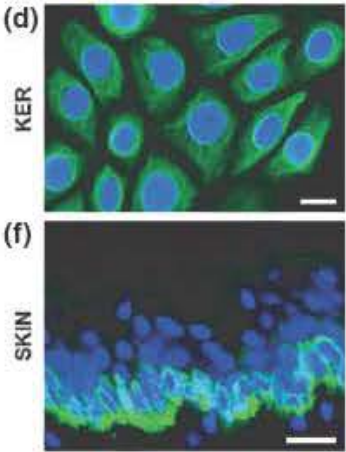

(g)

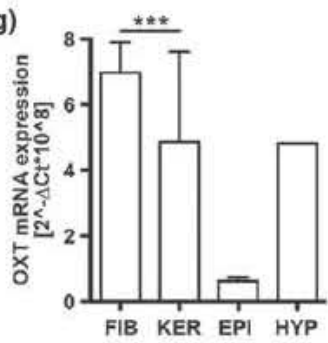

(h)
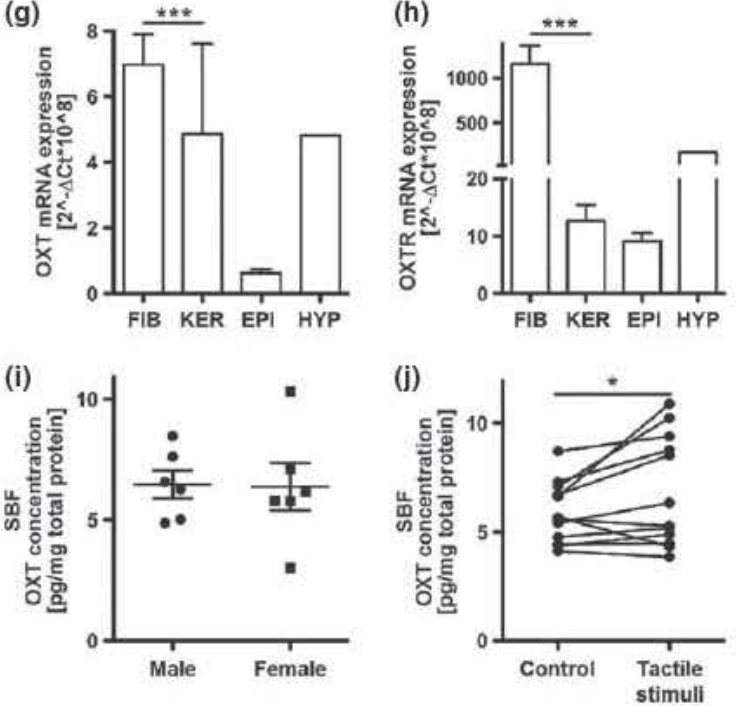

Figure 1. The oxytocin system is expressed in primary human skin celk. (a-f) Immunofluorescence localization of OXT (green) and the OXTR (green) was performed on dermal fibroblasts (FIB), keratinocytes (KER) and the epidermis of a skin section. Blue DAPI staining depicts nudei. Scale bar: $20 \mu \mathrm{m},(\mathrm{g}, \mathrm{h})$ Transcript levels for OXT and the OXTR in fibroblasts $(n=81)$, keratinocytes $(n=28)$, suction blister derived epidermis (EPI) $(n=12)$ and hypothalamus (HYP) $(n=1)$ were assessed by RT PCR. (i, j) OXT concentrations in human skin suction blister fluids (SBF) taken from both arms of male $(n=6)$ and female $(n=6)$ volunteers. (j) OXT concentrations in SBF derived from tactile stimulated and control areas. Wilcoxon signed rank test, ${ }^{*} p<0.05$; Mann Whitney $U$ test, ${ }^{* * *} p<0.001$

fluxes (3). In order to test, whether OXTR activation leads to increased $\mathrm{Ca}^{2+}$ fluxes in dermal fibroblasts and keratinocytes, we performed flow cytometry analyses. In both cell types, OXT led to a significant increase in $\mathrm{Ca}^{2+}$ currents in a dose dependent man ner, indicating that the OXTR is functional (Fig. 2a,c). Induction with $1 \mu \mathrm{M}$ OXT resulted in a twofold increase in $\mathrm{Ca}^{2+}$ currents compared to baseline. This effect was significantly inhibited by OXTR knockdown or by the OXTR antagonist L371,257. Visuali zation of OXT induced $\mathrm{Ca}^{2+}$ fluxes via fluorescence analyses confirmed OXTR signalling in dermal fibroblasts and keratinocytes (Fig. 2b,d). 

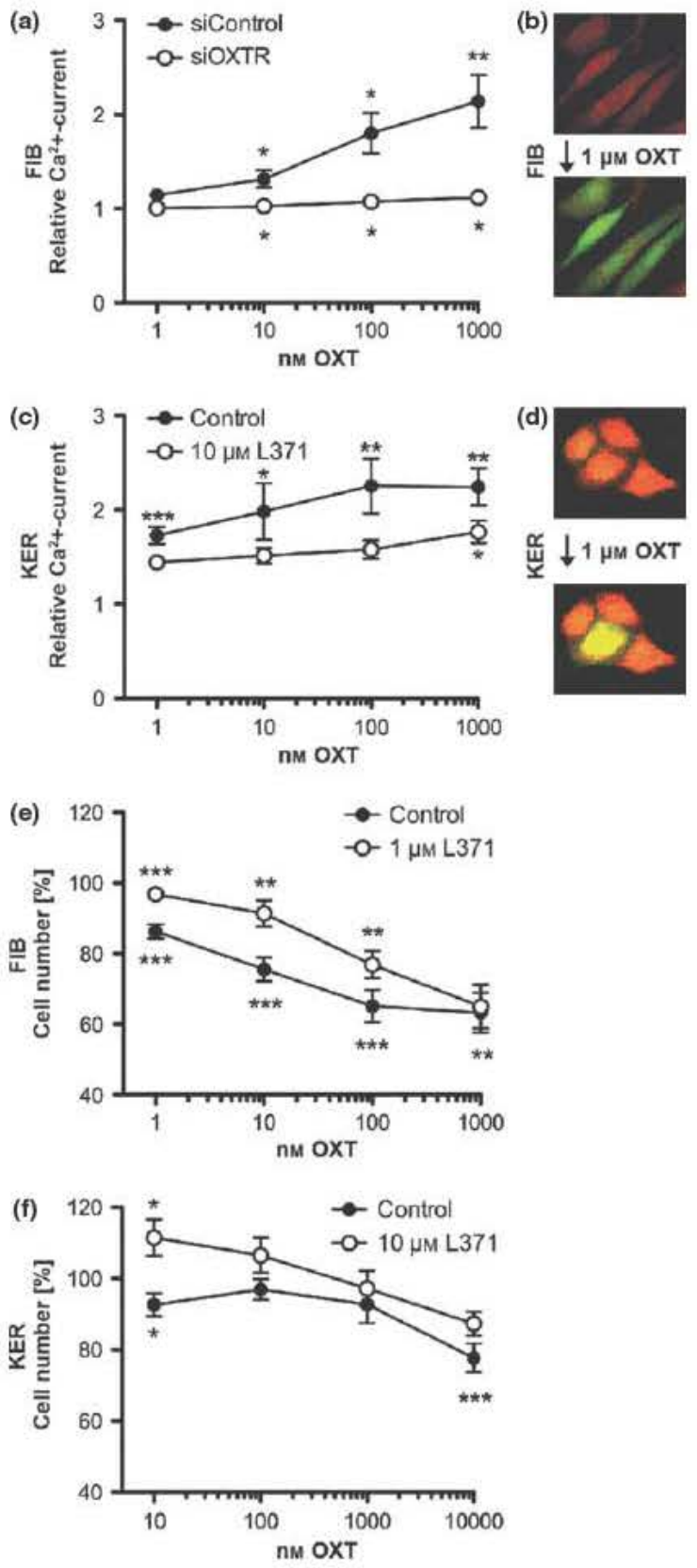

Figure 2. Oxytocin induces $\mathrm{Ca}^{2+}$ currents in skin cells and decreases thei proliferation rate. (a, c) OXT induced intracellular $\mathrm{Ca}^{2+}$ fluxes relative to baseline were measured in dermal fibroblasts (FIB) $(n=6)$ and keratinocytes (KER) $(n=6)$. OXTR knockdown in fibroblasts (siOXTR) and the treatment of keratinocytes with the OXTR inhibitor $L 371,257$ (L375) served as negative controls. (b, d)

Representative images of OXT induced $\mathrm{Ca}^{2+}$ fluxes (green). (e, $f$ ) Proliferation of OXT treated fibroblasts $(n=6)$ and keratinocytes $(n=6)$ relative to untreated control at day 7 (e) and day 4 (f). The OXTR inhibitor L 371 served as negative control. Asterisks show significance of OXT induced effects versus untreated or negative control. Paired $t$ test, ${ }^{*} P<0.05, * * P<0.01, * * * P<0.001$

Oxytocin decreases proliferation of dermal fibroblasts and keratinocytes

As OXT either promotes or inhibits cell growth of different cell types $(13,14)$, we investigated whether OXT modulates prolifera tion of skin cells.
Oxytocin treatment of fibroblasts and keratinocytes significantly decreased their proliferation rate (Fig. 2e,f). At day 7 of daily treatment with $1 \mathrm{nM}$ OXT, proliferation of fibroblasts was signifi cantly reduced ( $14 \pm 2 \%$ ) (Fig. $2 \mathrm{e}$ ). This growth inhibiting effect of OXT was dose dependently more pronounced using higher concentrations ( $1 \mu \mathrm{m}$ OXT: $37 \pm 6 \%$ decrease). Cotreatment with the OXTR antagonist L371,257 (1 $\mu \mathrm{M})$ counteracted the antiprolif erative effects of 1,10 and $100 \mathrm{~nm}$ OXT. In comparison to fibro blasts, keratinocytes responded to OXT treatment with a less pronounced but significant decrease in cell proliferation $(10 \mathrm{~nm}$ OXT: $7 \pm 3 \%, 10 \mu \mathrm{M}$ OXT: $22 \pm 4 \%$ ) (Fig. 2 f). Cotreatment with the OXTR antagonist L $371,257(10 \mu \mathrm{M})$ significantly antagonized the antiproliferative effect of OXT.

Oxytocin receptor knockdown induces oxidative stress, reduces glutathione levels and leads to enhanced pro inflammatory cytokine release of skin cells To elucidate OXTR mediated functions in the skin, we performed transient OXTR knockdown in dermal fibroblasts and keratinocytes.

Next, we investigated whether OXTR knockdown in skin cells affects their oxidative stress status. Determination of ROS levels revealed significantly increased intracellular ROS concentrations in OXTR depleted fibroblasts $(22 \pm 7 \%)$ and keratinocytes $(30 \pm 9 \%)$ (Fig. 3a). As expected, both cell types responded to UV irradiation with increased ROS formation (fibroblasts: $60 \pm 19 \%$, keratinocytes: $25 \pm 7 \%$ ). OXTR knockdown signifi cantly further aggravated the ROS status of irradiated skin cells (fibroblasts: $68 \pm 30 \%$, keratinocytes: $26 \pm 11 \%$ increase).

Additionally, we determined intracellular GSH concentrations to elucidate whether higher ROS levels correlate with lower GSH levels in OXTR depleted dermal fibroblasts and keratinocytes. In OXTR knockdown fibroblasts, GSH levels decreased significantly ( $38 \pm 5 \%$ ), whereas only a slight decrease was observed in OXTR knockdown keratinocytes (Fig. 3b). Irradiation of fibroblasts with $5 \mathrm{~J} / \mathrm{cm}^{2}$ UVA light diminished GSH concentrations significantly $(40 \pm 4 \%)$. Irradiation of keratinocytes with $100 \mathrm{~mJ} / \mathrm{cm}^{2}$ SSR light led to a marginal lowering of GSH level $(4 \pm 6 \%)$. Neverthe less, irradiated OXTR knockdown cells were more susceptible to GSH depletion than control cells. They exhibited a significant further decrease of GSH contents by approximately $13 \%$.

OXT modulates inflammatory processes $(19,31,49)$. In order to investigate whether the OXT system might be involved in the inflammatory stress response of the skin, we screened 27 cytokines concerning their regulation in OXTR knockdown skin cells. Out of these cytokines, the release of IL 6, CCL5 and CXCL10 was upregulated in keratinocytes in response to OXTR knockdown (Fig. 3c). The enhanced release was significant for all three cyto kines and the strongest effect was observed for CXCL10 (IL6: $164 \pm 65 \%$, CCL5: $226 \pm 90 \%, \quad$ CXCL10: $1297 \pm 632 \%$ ). In contrast, secretion of these cytokines was not modulated in OXTR depleted fibroblasts.

The oxytocin system is downregulated in atopic skin Our data revealed modulation of cytokine release, oxidative stress status and proliferation by the OXT system in skin cells (Figs 2e,f and 3). As these parameters are aberrant in $\operatorname{AD}(50,51)$, we explored whether the OXT system might be dysregulated in atopic skin. Thus, we analysed mRNA expression of OXT and its recep tor in dermal fibroblasts and keratinocytes derived from biopsies of healthy volunteers and atopic skin patients. RT PCR analyses 
(a)

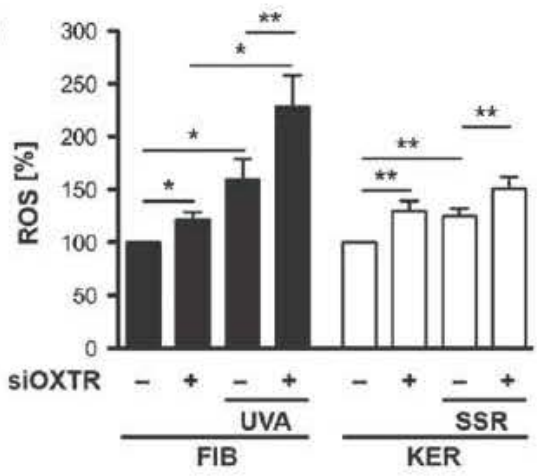

(b)

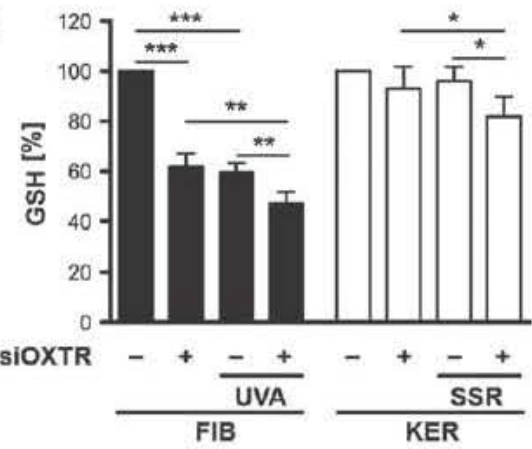

(c)

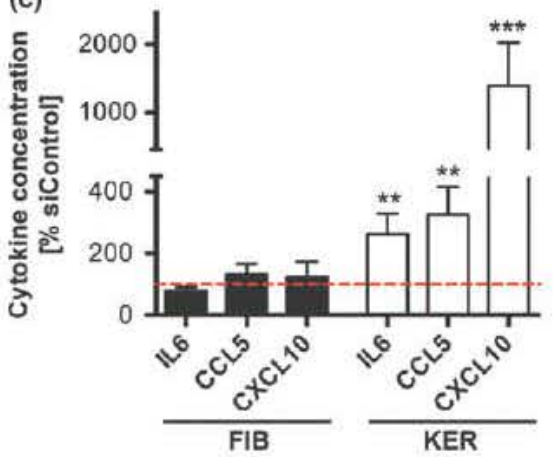

Figure 3. Oxytocin receptor knockdown leads to elevated concentrations of reactive oxygen species (ROS), reduced glutathione (GSH) levels and an increased release of pro inflammatory cytokines. (a-c) Primary human dermal fibroblasts (FIB) and keratinocytes (KER) were transfected with either scrambled (siControl) or OXTR specific (siOXTR) siRNA. (a) ROS levels in fibroblasts $(n=6)$ and keratinocytes $(n=12)$ were determined directly after sham irradiation and irradiation with $5 \mathrm{~J} /$ $\mathrm{cm}^{2}$ UVA or $100 \mathrm{ml} / \mathrm{cm}^{2}$ solar simulated light (SSR). (b) GSH levels in fibroblasts $(n=6)$ and keratinocytes $(n=6)$ were determined directly after sham irradiation and irradiation with $5 \mathrm{~J} / \mathrm{cm}^{2}$ UVA or $100 \mathrm{~mJ} / \mathrm{cm}^{2}$ SSR. (c) Cytokine concentrations in culture supernatants of OXTR knockdown fibroblasts $(n=4)$ and keratinocytes $(n=11)$ relative to siControl were determined. Supernatants were collected $24 \mathrm{~h}$ after addition of fresh media at day 4 post transfection. Paired $t$ test, $* P<0.05$, $* * P<0.01,{ }^{* * * P}<<0.001$

revealed significantly reduced OXT expression in atopic lesional fibroblasts $(2.8 \pm 2.6 \%)$ and in atopic peri lesional keratinocytes $(0.9 \pm 0.1 \%)$ compared to healthy controls (Fig. 4a). Atopic peri lesional fibroblasts and atopic lesional keratinocytes also showed reduced OXT expression. Additionally, OXTR expression was significantly decreased in both, atopic peri lesional and lesional fibroblasts ( $620 \pm 52 \%, 560 \pm 65 \%$, respectively), compared to healthy fibroblasts (Fig. 4b). Atopic keratinocytes also showed a decreased OXTR expression (Fig. 4b). Accordingly, a similar trend to reduced OXTR expression levels was detected in epidermis
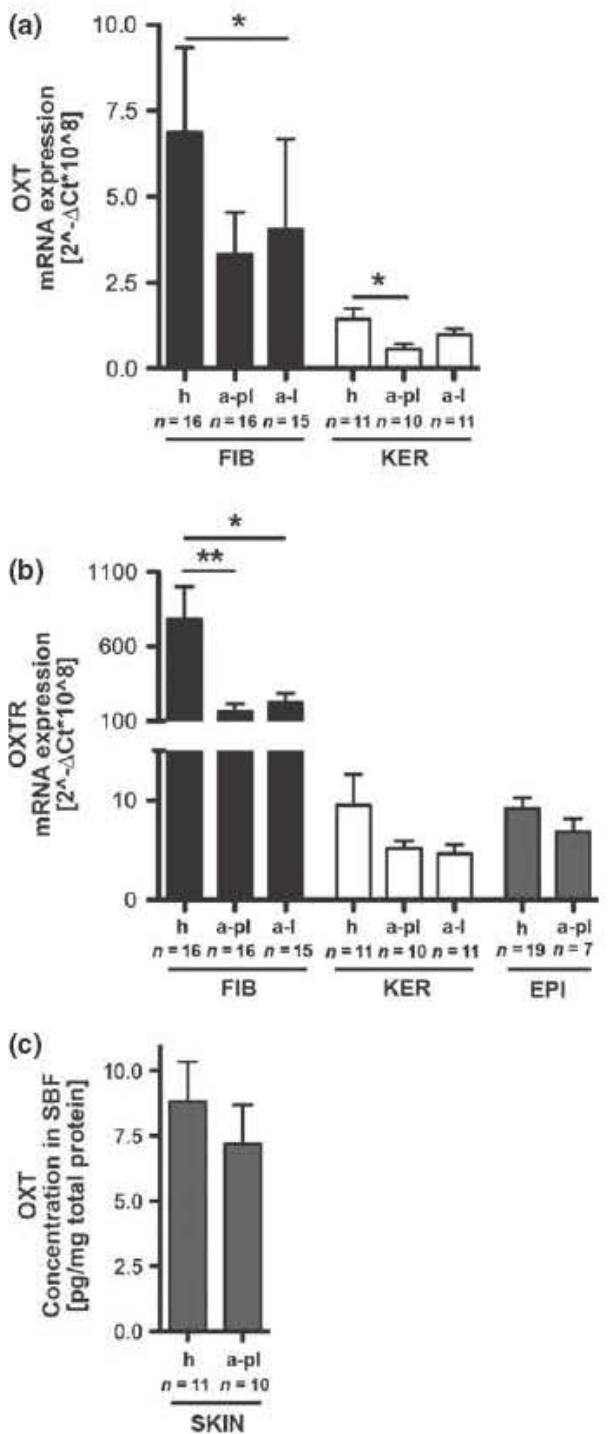

Figure 4. Expression of oxytocin and oxytocin receptor is reduced in atopic skin. (a, b) Transcript levels for OXT and its receptor in healthy $(\mathrm{h})$, peri lesional ( $\mathrm{pl}$ ) and lesional (I) atopic (a) dermal fibroblasts (FIB), keratinocytes (KER) and suction blister derived epidermis (EPI) were assessed by RT PCR. (C) OXT concentrations in suction blister fluids (SBF) of peri lesional atopic and healthy skin were detected. Unpaired $t$ test, Mann-Whitney $U$ test, ${ }^{*} P<0.05, * * P<0.01$.

derived from atopic peri lesional suction blisters (Fig. 4b). To evaluate OXT levels in atopic versus healthy skin in vivo, we measured OXT concentrations in suction blister fluids. Suction blister fluids derived from peri lesional atopic skin exhibited a slight decrease of OXT concentration compared to the healthy control (Fig. 4c). These data suggest that atopic skin displays a deficit in OXT signalling.

\section{Discussion}

The neurohypophyseal hormone OXT exerts a wide spectrum of central and peripheral effects, including facilitation of reproduc tion at several levels, establishment of complex social behaviour, neuroendocrine modulation and immunological processes $(3,31)$. Apart from studies, investigating the impact of subcutaneously injected OXT on different inflammation models $(29,30)$, the role of the OXT system in skin has not been described so far. Recently, Denda 
et al. (38) demonstrated that OXT is expressed in human skin kerati nocytes and released in response to calcium influx via P2X receptors.

In this study, we showed that OXT is expressed not only in keratinocytes, but also in human skin derived dermal fibroblasts. Moreover, we were able to detect mRNA transcripts of the OXTR in dermal fibroblasts and keratinocytes. A previous study reported OXTR expression in human foreskin fibroblasts (52). Our immu nohistochemical stainings of OXT and its receptor in human skin showed an expression of OXT in all epidermal layers and a prefer ential expression of the OXTR in the basal layers. Denda et al. (38) also found OXT immunoreactivity throughout the epidermis.

In a study with 12 healthy volunteers, we showed that tactile stimulation of the skin increases OXT concentrations in suction blister fluids. In contrast to former studies reporting on elevated systemic OXT levels after partner hugs in humans (53) or repeated massage like stroking in rats (54), our observation demonstrates local cutaneous OXT release in vivo. In anaesthetized rats, plasma OXT levels rise in response to touch or vagal and sciatic nerve activation proposed to originate from peripheral nerves in the skin (55). With regard to our findings of cutaneous OXT expression, the release might also originate from skin cells such as dermal fi broblasts and keratinocytes.

Stimulation of dermal fibroblasts and keratinocytes with OXT induced an increase in intracellular $\mathrm{Ca}^{2+}$ demonstrating the functionality of the OXTR in these skin cells. The specificity of this response was confirmed by either blocking $\mathrm{Ca}^{2+}$ signalling via OXTR knockdown or application of the OXTR antagonist L371,257. In smooth muscle cells, activation of the OXTR also induces $\mathrm{G}_{\mathrm{q}}$ mediated increases in $\mathrm{Ca}^{2+}$ leading to contraction (56).

Depending on cell type, the activation of the OXTR is either associated with stimulation $(14,57)$ or inhibition $(11,44)$ of proliferation. We found a suppressive effect of OXT on the prolif eration of dermal fibroblasts and keratinocytes. The regulation of proliferation by OXT is complex. It depends on expression levels and coupling of $\mathrm{G}_{\mathrm{q}}$ and $\mathrm{G}_{\mathrm{i}}$ protein isoforms, OXT concentration and OXTR recruitment to caveolae and cholesterol content in the cell membrane $(15,44,58)$. The regulatory mechanisms underlying the modulation of proliferation by OXT signalling in dermal fibroblasts and keratinocytes need to be elucidated in future studies.

By inhibiting OXTR signalling, our data revealed an impact of OXT on the modulation of oxidative stress, intracellular GSH levels and cytokine release in dermal fibroblasts and keratinocytes. OXTR knockdown skin cells showed increased susceptibility to oxidative stress. This might indicate a cytoprotective role of OXT with regard to oxidative stress, as the formation of ROS leads to damage to nucleic acids, proteins and membrane lipids (59). OXT has anti oxi dative activity by scavenging radicals and preventing the oxidation of lipoproteins (60). Furthermore, OXT improves the anti oxidative state of colonic tissue in a colitis model in rat (49) and balances ROS with cardioprotective efficacy in ischaemic rat heart (61). ROS formation is closely related to a decrease in GSH levels (62), thereby reducing the cellular anti oxidative defense $(63,64)$. Consistent with higher ROS levels in OXTR knockdown dermal fibroblasts and keratinocytes, these cells also displayed decreased intracellular GSH concentrations. Sepsis induced pelvic inflammation is also associ ated with increased ROS and decreased GSH levels. OXT adminis tration prevents the depletion of tissue GSH in this inflammatory condition (27). The treatment of pregnant rats with the OXTR antagonist atosiban elevates oxidative stress in the hearts of the new borns (65). In keratinocytes, the effects of OXTR knockdown on ROS formation and GSH levels were less pronounced than in der mal fibroblasts, indicating a cell type specific susceptibility of skin cells to oxidative stress and its modulation by OXT. In accordance with this finding, Marionnet et al. (66) reported a higher sensitivity to oxidative stress of dermal fibroblasts in comparison with kerati nocytes in UV irradiated skin models.

In contrast to dermal fibroblasts, keratinocytes released larger quantities of the cytokines IL6, CCL5 and CXCL10 in response to OXTR knockdown, thus confirming a cell type differential response to impaired OXT signalling. OXT has been shown to decrease the release of interleukins and other inflammatory mediators in various tissues and on a systemic level $(19,31,49)$. In myometrial cells, OXT treatment reduces CCL5, CXCL5 and CCL20 expression (67). Administration of OXT in humans reduces endotoxin induced increases in plasma cytokines amongst others IL6 and CXCL10 (68).

The impact of OXT on the modulation of oxidative and inflam matory stress responses in skin cells suggests a role in chronic inflammatory skin conditions such as AD. Actually, OXT and its receptor were downregulated in dermal fibroblasts and keratino cytes derived from atopic skin. In a translational approach, we confirmed the aberrant expression of the OXT system in atopic skin as suction blister derived epidermis and fluids from $\mathrm{AD}$ patients displayed decreased OXTR and OXT levels.

The dynamic modulation of OXT and OXTR expression has been demonstrated in various studies: The OXT system is highly regulated during gestation, parturition and lactation, thus enabling circulating OXT to target a particular organ depending on the precisely regulated tissue specific expression of the OXTR $(69,70)$. Furthermore, the OXTR is regulated by oestrogen, foetal bovine serum and lysophospholipids in vitro $(71,72)$. The OXTR pro moter also displays several potential interleukin response elements (73) explaining the negative regulation of OXTR gene expression by IL6, IL1 $\beta$ and interferon $(7,8)$. Sepsis, which is associated with massive production of inflammatory mediators and neuroendo crine alterations, decreases OXT gene expression in the brain (74). As the skin of $\mathrm{AD}$ patients is chronically inflamed, displaying a plethora of up regulated cytokines (75 77), elevated levels of inflammatory mediators might account for the decreased OXT and OXTR gene expression in atopic skin. As a consequence, defi cits in OXT signalling possibly enhance oxidative stress and inflammation in atopic skin resulting in a vicious circle.

Altogether, we identified the OXT system as a novel neuroen docrine mediator of skin cell proliferation and stress responses in vitro. We speculate that the OXT system is also involved in skin neuroendocrine networks. Cutaneous CRH/POMC system activity is, amongst others, determined by cytokine release and is proposed to form a function analogous to the HPA axis $(23,25,78)$. As OXT plays a role in modulating the HPA axis (79 81) and CRH signal ling in term 'myometrium' (24), it is tempting to suggest that OXT might also interact with the skin's local neuroendocrine mediators, such as CRH. Moreover, the OXT promoter can be stimulated by oestrogen receptors and thyroid hormone receptors $(82,83)$, which are also produced by skin cells $(21,8486)$. In line with the finding that the skin shares numerous mediators with the CNS (22), OXT turns out to be a new candidate of a brain and skin derived neuropeptide. Additionally, as we were able to dem 
onstrate OXTR expression by skin cells, either skin cell derived or plasma derived OXT might signal in an autocrine as well as in a paracrine way in the skin. This would link into the hypothesized bidirectional brain skin connection $(22,87)$.

In summary, the OXT system was characterized in human skin demonstrating its relevance in skin physiology. Dermal fibroblasts and keratinocytes responded to OXT via $\mathrm{Ca}^{2+}$ influx, thus proving functionality of the OXTR in skin cells. OXTR knockdown via siRNA or inhibition by a specific antagonist revealed an impact of OXT on the modulation of proliferation, ROS formation, GSH content and cytokine release in dermal fibroblasts and keratino cytes. The effects of OXT signalling on skin cell physiology point to a role of OXT in cutaneous inflammation. Indeed, we found a reduced expression of the OXT system in $\mathrm{AD}$ skin indicating its clinical relevance to chronic inflammatory skin diseases.

\section{Author contribution}

V. D., J. K., F. S. and G. N. designed the research. V. D. and A. G. performed the research. V. D and G. N. analysed the data. F. S. and H. W. contributed essential reagents and tools. V. D. and D. R. wrote the paper. A. B. and G. N. critically revised the manuscript. All authors have approved the submitted and final version of the manuscript and confirm that the submitted work complies with the ethical policies of Experimental Dermatology.

\section{Conflict of interests}

The authors have declared no conflicting interests.

\section{References}

1 Kirsch P. Esslinger C, Chen Q et al. I Neurosci 2005: 25: 11489-11493.

2 Ferguson J N, Young L J, Hearn E F et al. Nat Genet 2000: 25: 284-288.

3 Gimpl G, Fahrenholz F. Physiol Rev 2001: 81: 629-683.

4 Gutkowska J, Jankowski M. Pharmaceuticals 2009: 2: 168-183.

5 Brownstein M J, Russell J T, Gainer H. Science 1980: 207: 373-378.

6 Vrachnis N, Malamas F M, Sifakis S et al. Int J Endocrinol 2011: 2011: 350546.

7 Ivell R, Bathgate R A, Walther $\mathrm{N}$ et al. Adv Exp Med Biol 1998: 449: 297-306.

8 Schmid B, Wong S, Mitchell B F. Endocrinology 2001: 142: 1380-1385.

9 Peter J, Burbach H, Adan R A et al. Cell Mol Neurobiol 1995: 15: 573-595

10 Verbalis J G. Front Neuroendocrinol 1999: 20: 146-156.

11 Rimoldi V, Reversi A, Taverna E et al. Oncogene 2003: 22: 6054-6060.

12 Zhou X B, Lutz S, Steffens F et al. Mol Endocri nol 2007: 21: 740-752

13 Cassoni P, Marrocco T, Bussolati B et al. Mol Cancer Res 2006: 4: 351-359.

14 Cassoni P, Sapino A, Marrocco T et al. J Neuro endocrinol 2004: 16: 362-364.

15 Reversi A, Cassoni P, Chini B. J Mammary Gland Biol Neoplasia 2005: 10: 221-229.

16 Cassoni P, Sapino A, Stella A et al. Adv Exp Med Biol 1998: 449: 245-246.

17 Bakos J, Strbak V, Ratulovska N et al. Cell Mol Neurobiol 2012: 32: 891-896.

18 Amico J A, Mantella R C, Vollmer R R et al. J Neuroendocrinol 2004: 16: 319-324.

19 Ivell R, Russell J A. Rev Reprod 1996: 1: 13-18.

20 Neumann | D. Prog Brain Res 2002: 139 147-162

21 Slominski A, Wortsman J. Endocr Rev 2000: 21: 457-487.

22 Slominski A T, Zmijewski M A, Skobowiat C et al. Adv Anat Embryol Cell Biol 2012: 212: v, vii, $1-115$

23 Slominski A, Wortsman J, Luger T et al. Physiol Rev 2000: 80: 979-1020.

24 Grammatopoulos D K, Hillhouse E W. Endocri nology 1999: 140: 585-594

25 Slominski A, Pisarchik A, Tobin D J et al. Endo crinology 2004: 145: 941-950.

26 Slominski A, Ermak G, Hwang J et al. FEBS Lett 1995: 374: 113-116.

27 Iseri S O, Sener G, Saglam B et al. I Surg Res 2005: 126: 73-81.

28 Vitalo A, Fricchione J, Casali $\mathrm{M}$ et al. PLoS ONE 2009: 4: e5523.

29 Iseri S O, Gedik I E, Erzik C et al. Burns 2008: 34: 361-369.

30 Padhy B M, Kumar V L. Mediators Inflamm 2005: 2005: 360-365.
31 Szeto A, Nation D A Mendez A J et al Am Physiol Endocrinol Metab 2008: 295: E1495E1501.

32 De Benedetto A, Agnihothri R, McGirt L $Y$ et al. J Invest Dermatol 2009: 129: 14-30.

33 Proksch E, Folster Holst R, Jensen J M. J Derma tol Sci 2006: 43: 159-169.

34 Valyi Nagy I, Jensen P J, Albelda S M et al. J Invest Dermatol 1992: 99: 350-356.

35 Matsuura H, Sakaue M, Subbaramaiah K et al. Biol Chem 1999: 274: 29138-29148.

36 Mascia F, Mariani V, Girolomoni G et al. Am Pathol 2003: 163: 303-312.

37 Giustizieri M L, Mascia F, Frezzolini A et al. Allergy Clin Immunol 2001: 107: 871-877.

38 Denda S, Takei K, Kumamoto J et al. Exp Der matol 2012: 21: 535-537.

39 Kiistala U. J Invest Dermatol 1968: 50: 129-137.

40 Roggenkamp D, Falkner S, Stab F et al. J Inves Dermatol 2012: 132: 1892-1900.

41 Amico J A, Seif S M, Robinson A G. J Clin Endo crinol Metab 1981: 52: 988-993.

42 Paredes J, Szeto A, Levine J E et al. Psychoneu roendocrinology 2006: 31: 1062-1075.

43 Eriksson M, Uvnas Moberg K. Neuroendocrinol ogy 1990: 51: 237-240.

44 Guzzi F, Zanchetta D, Cassoni $P$ et al. Oncogene 2002: 21: 1658-1667.

45 Novak E J, Rabinovitch P S. Cytometry 1994: 17 135-141.

46 Dengler W A, Schulte J, Berger D P et al. Anti cancer Drugs 1995: 6: 522-532.

47 Izykowska I, Cegielski M, Gebarowska E et al. In Vivo 2009: 23: 739-745.

48 Holt Lunstad J, Birmingham W A, Light K C. Psychosom Med 2008: 70: 976-985.

49 Iseri S O, Sener G, Saglam B et al. Peptides 2005: 26: 483-491.

50 Tsuboi H, Kouda K, Takeuchi $\mathrm{H}$ et al. Br J Der matol 1998: 138: 1033-1035

51 Tsukahara H, Shibata R, Ohshima $Y$ et al. Life Sci 2003: 72: 2509-2516.

52 Kinsey C G, Bussolati G, Bosco $M$ et al. J Cell Mol Med 2007: 11: 96-110.

53 Light K C, Grewen K M, Amico J A. Biol Psycho 2005: 69: 5-21

54 Lund I, Ge Y, Yu L C et al. Eur J Neurosci 2002 16: $330-338$

55 Stock S, Uvnas Moberg K. Acta Physiol Scand 1988: 132: 29-34

56 Yazawa $\mathrm{H}$, Hirasawa A, Horie $\mathrm{K}$ et al. Br J Phar macol 1996: 117: 799-804

57 Tom N, Assinder S J. Int J Biochem Cell Biol 2010: 42: 202-205.

58 Busnelli M, Sauliere A, Manning M et al. J Bio Chem 2012: 287: 3617-3629.

59 Girard P M, Pozzebon M, Delacote F et al. DNA Repair (Amst) 2008: 7: 1500-1516.

60 Moosmann B, Behl C. Mol Pharmacol 2002: 61 260-268.
61 Faghihi $M$, Alizadeh $A M$, Khori $V$ et al. Peptides 2012: 37: 314-319.

62 Moysan A, Marquis I, Gaboriau F et al. J Invest Dermatol 1993: 100: 692-698.

63 Lu S C. Mol Aspects Med 2009: 30: 42-59.

64 Valko M, Leibfritz D, Moncol J et al. Int J Biochem Cell Biol 2007: 39: 44-84.

65 Simsek Y, Celik O, Karaer A et al. Arch Gynecol Obstet 2012: 285: 655-661.

66 Marionnet C, Pierrard C, Lejeune F et al. PLoS ONE 2010: 5: e12059.

67 Hua R, Pease J E, Sooranna S R et al. Endocri nology 2012: 153: 481-491.

68 Clodi M, Vila G, Geyeregger R et al. Am J Phys iol Endocrinol Metab 2008: 295: E686-E691.

69 Soloff M S, Alexandrova M, Fernstrom M J. Sci ence 1979: 204: 1313-1315.

70 Breton C, Di Scala Guenot D, Zingg H H. J Mol Endocrinol 2001: 27: 175-189.

71 Bale T L, Dorsa D M. Endocrinology 1997: 138: 1151-1158.

72 Jeng $Y$ J, Soloff $S \mathrm{~L}$, Anderson G D et al. Endo crinology 2003: 144: 61-68.

73 Inoue $T$, Kimura $T$, Azuma $C$ et al. J Biol Chem 1994: 269: 32451-32456.

74 Oliveira Pelegrin G R, Aguila F A, Basso P J et al. Peptides 2010: 31: 1847-1852.

75 Gittler J K, Shemer A, Suarez Farinas M et al. J Allergy Clin Immunol 2012: 130: 1344-1354.

76 Raap U, Weissmantel S, Gehring $M$ et al. Pediatr Allergy Immunol 2012: 23: 285-288.

77 Nomura I, Goleva E, Howell M D et al. J Immu nol 2003: 171: 3262-3269.

78 Slominski A, Wortsman J, Tuckey R C et al. Mol Cell Endocrinol 2007: 265-266: 143-149.

79 Windle R J, Shanks N, Lightman S L et al. Endo crinology 1997: 138: 2829-2834.

80 Neumann I D, Torner L, Wigger A. Neuroscience 2000: 95: 567-575.

81 Neumann I D, Wigger A, Torner $L$ et al. J Neuro endocrinol 2000: 12: 235-243.

82 Adan R A, Cox J J, Beischlag $T$ V et al. Mol Endocrinol 1993: 7: 47-57.

83 Richard S, Zingg H H. J Biol Chem 1990: 265 : 6098-6103.

84 Torma H, Rollman O, Vahlquist A. Acta Derm Venereol 1993: 73: 102-107.

85 Hasselquist M B, Goldberg N, Schroeter A et al. J Clin Endocrinol Metab 1980: 50: 76-82.

86 Punnonen R, Lovgren T, Kouvonen I. J Endocri nol Invest 1980: 3: 217-221.

87 Arck P C, Slominski A, Theoharides T C et al. J Invest Dermatol 2006: 126: 1697-1704. 